\title{
Comparative evaluation of pathophysiology of type 1 diabetes and gestational diabetes mellitus: A review
}

\author{
Amisha Sharma, Priyanka and Amarish Kumar Sharma \\ Department of Biosciences and Bioengineering, Lovely Professional University, Phagwara- 144411, Punjab, India
}

\section{Article Info}

\section{Article history}

Received 8 April 2021

Revised 28 May 2021

Accepted 29 May 2021

Published online 30 June 2021

\section{Keywords}

Gestational diabetes mellitus

Diabetes

Obesity

Pregnancy complications

Screening

Pathophysiology

\begin{abstract}
Diabetes is a chronic and progressive metabolic disorder characterized by a high level of blood sugar, i.e., glucose in the body. Glucose is the main source of energy in the human body. The level of glucose in the blood is controlled by the hormone called insulin, released by the pancreas. Gestational diabetes mellitus (GDM) is characterized as glucose intolerance that begins and first recognition during pregnancy. Diabetes is very common in Asian countries, especially Indians. Obesity and advanced gestational age are the other risk factors for gestational diabetes.The acute, long-term, and transgenerational health complications of diabetes disease affect approximately 9-25 per cent of pregnancies worldwide. The present article discusses the concept, risk factors, pathogenesis, screening, and treatment of diabetes in pregnancy, as well as the complications, it can cause the mother and the fetus, as well as the long-term consequences.
\end{abstract}

\section{Introduction}

Diabetes is not a modern phenomenon. Diabetes mellitus has been known for centuries ago by the ancient Romans, Indians, Arabs, and the Greeks. In India, it was called madhumeha, meaning honey urine. While in Greece, they named it siphon, which is a reference to increased urination, an important sign of diabetes today. The discovery of the pancreas in diabetes was made by JosephVon Mering and Oskar Minkowski in 1889.

Diabetes mellitus is a complex and metabolic disorder in which the level of glucose in the blood is increased and the body is unable to produce a sufficient amount of insulin (Maritim et al., 2003). Insulin is the polypeptide hormone synthesized in the beta cells of the pancreas and functions as the main glucose regulator. It communicates with the liver as well as muscle and adipose tissue (Joshi et al., 2007). In diabetes, the body is not producing enough of the hormone insulin and because of the lack of insulin, the blood levels of sugar increase. Now, the high level of sugar in the bloodstream slowly poisons parts of the body such as the eyes, heart, nerves, liver, and blood flow in feet and fingers which may cause heart stroke, neuropathy, weight loss, and urination (Wallace, 2004).

Diabetes mellitus is classified into four different types as follow (American Diabetes Association, 2004):

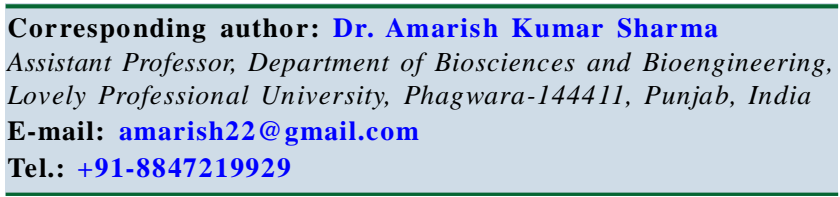

Copyright () 2021 Ukaaz Publications. All rights reserved.

Email: ukaaz@yahoo.com; Website: www.ukaazpublications.com i. Type 1 diabetes: Type 1 diabetes is autoimmune insulindependent in which certain areas of the endocrine portion of the pancreas are destroyed which may lead to insulin deficiency.

ii. Idiopathic diabetes: Idiopathic diabetes is another type of type 1 diabetes with unknown etiologies and is fully inherited.

iii. Type 2 diabetes: Type 2 diabetes is non-insulin-dependent which occurs due to insulin secretory defect and insulin resistance. Here, body cells are non-responding to insulin as they should or the gene of insulin gets mutated.This form was previously known as "non-insulin-dependent diabetes mellitus" (NIDDM) or "adult-onset diabetes".

iv. Gestational diabetes: Gestational diabetes is the third main form and occurs when pregnant women without a previous history of diabetes develop a high blood glucose level. It is any degree of glucose intolerance that begins or first recognition during pregnancy.

Diabetes is becoming increasingly common among people with a normal or underweight body mass index which is BMI. And, it is no longer a disease most prevalent in urban areas. This means that even though diabetes will rise in urban areas, it will rise even more steeply in rural areas in developing countries. We are currently witnessing an increasing number of people in rural areas in both low and middle-income countries who will suffer from diabetes. Gestational diabetes mellitus (GDM) is a chronic illness in which the blood sugar levels rise in women during pregnancy. This causes a lot of short-term and long-term risk factors for both mother and the unborn baby. Glucose sensitivity in varying degrees with initiation or first identification during pregnancy is known as Gestational Mellitus. 


\section{Risk factors for GDM}

Over the years, there are several risk factors observed during the epidemiological studies of GDM. These risk factors conclude age, ethnicity, obesity, family history of type 2 diabetes, previous history of GDM.

i. Age: Advanced maternal age is an established risk factor regarding GDM. It is shown in various studies in the USA, that women > 40 have more risk of GDM than women who are $<30$. Considering that the average age of childbearing has been increasing over the past 4 decades, it is understandable as to why there a high risk for advanced maternal age. The studies have also shown women age $<20$ have a lower risk of GDM. It is also said that as the age of women progresses, it can also lead to an increase in mental stress which can result in anxiety and that can activate the sympathetic nerve of the hypothalamic-pituitary-adrenal axis which can further lead to the rise in blood glucose (Solomon et al., 1997).

ii. Ethnicity: The variability in the prevalence of GDM has still been observed even if, the same diagnostic strategies were used. So, it was observed that the variations might be because of the different ethnic backgrounds within and between different countries.

Countries like Australia, the USA, Canada which have a vast variety of ethnic population, the variations of prevalence in GDM was observed between different ethnicities such as in Northern California non-Hispanic whites (4.5\%) and African American $(4.4 \%)$ showed the lowest GDM prevalence in comparison to Philippines $(10.9 \%)$ that showed the highest prevalence followed by Asians (10.2\%) (Hedderson et al., 2012). Also in Australia, it was seen that South Asian women showed more risk of GDM than women of Australian and New Zealand origin. It can also see in studies that South Asian women have a high prevalence of GDM and this difference might be because of different lifestyles (Anna et al., 2008).

iii. BMI: BMI commonly indicates the degree of obesity. Obesity is the main driver of diabetes. Pre-pregnancy BMI was due to the occurrence of GDM. The overweight and obese women were prone to GDM due to the strong insulin resistance. It was observed in studies that women with GDM have high BMI than women in the non-GDM group and the risk of GDM was also increased by 0.28 folds at each additional BMI of $1 \mathrm{~kg}$ per meter square. That is why physical activities during and before pregnancy lower the risk of GDM (Zhao et al., 2018).

\subsection{Risk factors for Type 1 diabetes}

i. Genetic factors: Type 1 diabetes is normally present in people without a family ancestry. Just $10-15 \%$ of the patients have a first-or second-degree relative with the infection. Nonetheless, the lifetime hazard for creating T1DM is essentially expanded (Beyan et al., 2012). The primary qualities inclining to T1DM are inside the significant histocompatibility complex (MHC) district, frequently called HLA (human leukocyte antigen) and situated on chromosome 6. HLA complex polymorphic alleles are liable for $40-50 \%$ of the hereditary risk of T1DM development (Aly et al., 2006). ii. Major histocompatibility complex (MHC): MHC is a group of genes that code for proteins found on the surfaces of cells that help the immune system recognize foreign substances. Human leukocyte antigen (HLA) gene loci are recognized into two classes. Class I HLA molecules are related to antigens that are perceived by the receptor of cytotoxic T lymphocytes $(\mathrm{CD} 8+)$, which at last obliterate the antigenic objective and are communicated by most cells. Class II HLA particles are fundamental for the acknowledgment of antigens by $\mathrm{T}$ aide lymphocytes $(\mathrm{CD} 4+)$, which start the immune response and advance cellular co-activity and are communicated simply by immune cells.

iii. Insulin variable number tandem repeat (VNTR): Variable number tandem repeat (VNTR) are short nucleotide sequences, i.e., 20-100 base pairs that vary in copy number in bacterial genomes. Polymorphisms in the locale of the insulin gene promoter are assessed to represent about $10 \%$ of the hereditary inclination for T1DM. This region is situated on the short arm of chromosome 11 and is near to the gene encoding for proinsulin. This polymorphic site comprises a variable number of tandem repeats (VNTRs). It is classified into two common classes: the small class (26-63 repeats, type I) or the large class (140-243 repeats, type III) (Barratt et al., 2004).

iv. Environmental factors: Environmental factors also play a significant role in the pathogenesis of T1DM. Strong evidence for this derives from the study of monozygotic twins, where the event of the disease in both siblings varies around $50 \%$ and never arrives at $100 \%$. The environmental factors include viruses (rubella, enteroviruses), toxins (Beyan et al., 2012).

v. Viral infection: Viruses address significant triggers for T1DM pathogenesis and this was initially depicted by epidemiological perceptions. Children uncovered during fetal life to rubella have an increased occurrence of T1DM, besides other autoimmune disorders, such as autoimmune thyroiditis (Jaïdane et al., 2010). Moreover, RNA or proteins from such viruses have been identified in the pancreas and other adjoining tissues from patients with T1DM. There is likewise a connection between T1DM and the interferon-induced helicase 1 (IFIH1) gene, which encodes for the MDA5. Upon viral infection, MDA5 acts as a cytoplasmic sensor and stimulates the production of molecular mediators of the innate immune response (Hober et al., 2010).

\section{Etiology of gestational diabetes mellitus}

Gestational diabetes mellitus is a progressing health issue. As per IDF, the most recent prevalence estimates (2017) is that GDM represents $86.4 \%$ of all hyperglycemia in pregnancy. However, this prevalence broadly shifts because of different population characteristics and different diagnostic and screening criteria. In 2010 , the assessed prevalence of the USA was estimated to be $4.6 \%$ 9.2\% yet utilizing IADPSG criteria in hyperglycemia and Neonatal result studies the frequency of GDM was $17.8 \%$. The general predominance has been expanded in recent years due to using different diagnostic criteria. Diabetes is on the rise, no longer a disease of predominantly rich nations. The prevalence of diabetes is steadily increasing everywhere, most markedly in the world's middle-income countries. The latest figures from 2012 show that diabetes caused the death of 1.5 million people and led to an additional 2.2 million deaths caused by higher than optimal blood 
glucose which increases the risk of cardiovascular and other diseases. $43 \%$ of these 3.7 million deaths happened before individuals reached 70 years of age. Diabetes is probably recognized as one of the biggest global health challenges in the 21 st century. And according to the global report on diabetes it is estimated that 422 million adults were living with diabetes in 2014. These numbers equal a fourfold increase since 1980 or arise from $4.7 \%$ to $8.5 \%$ in the adult population affected. The prevalence is rising in all income groups. However, over the past decade, it has risen faster in low and middleincome countries rather than in high-income countries. WHO divides the world into six regions, the Americas, Europe, Eastern Mediterranean, Africa, Southeast Asia, and Western Pacific. The largest number of people with diabetes were estimated to reside in Southeast Asia and Western Pacific regions. In total, three-quarters of people living with diabetes live in low and middle-income countries and most of them have only limited access to treatment.
According to WHO, it is analyzed that the prevalence of diabetes in the eastern Mediterranean region was the highest, and the prevalence of diabetes in the African region and the European region was the lowest. The Middle East and some North African countries have the highest prevalence of GDM at $8.8-20 \%$, followed by South-East Asia at 9.6-18.3\%, the Western Pacific region at 4.5-20.3\%, South and Central America at 7.1-16.6\%, Sub-Saharan Africa at 8.5-13.1\%, and North America and the Caribbean at $8.5-13.1 \%$ ranging from $6.5-11.9 \%$. The minimal prevalence of GDM is seen in Europe ranging from $1.8-31 \%$. But, variations are as yet seen in prevalence within and between countries (Figure 1) (Zhu and Zhang et al., 2016). Based on studies from 200 countries and a total of 19.2 million participants, the number of obese men to the left and women to the right has increased ever since 1975 until 2014. If, the curve continues its steep upward climb, roughly a fifth of men, $18 \%$, and women, $21 \%$ worldwide, will be obese by 2025 .

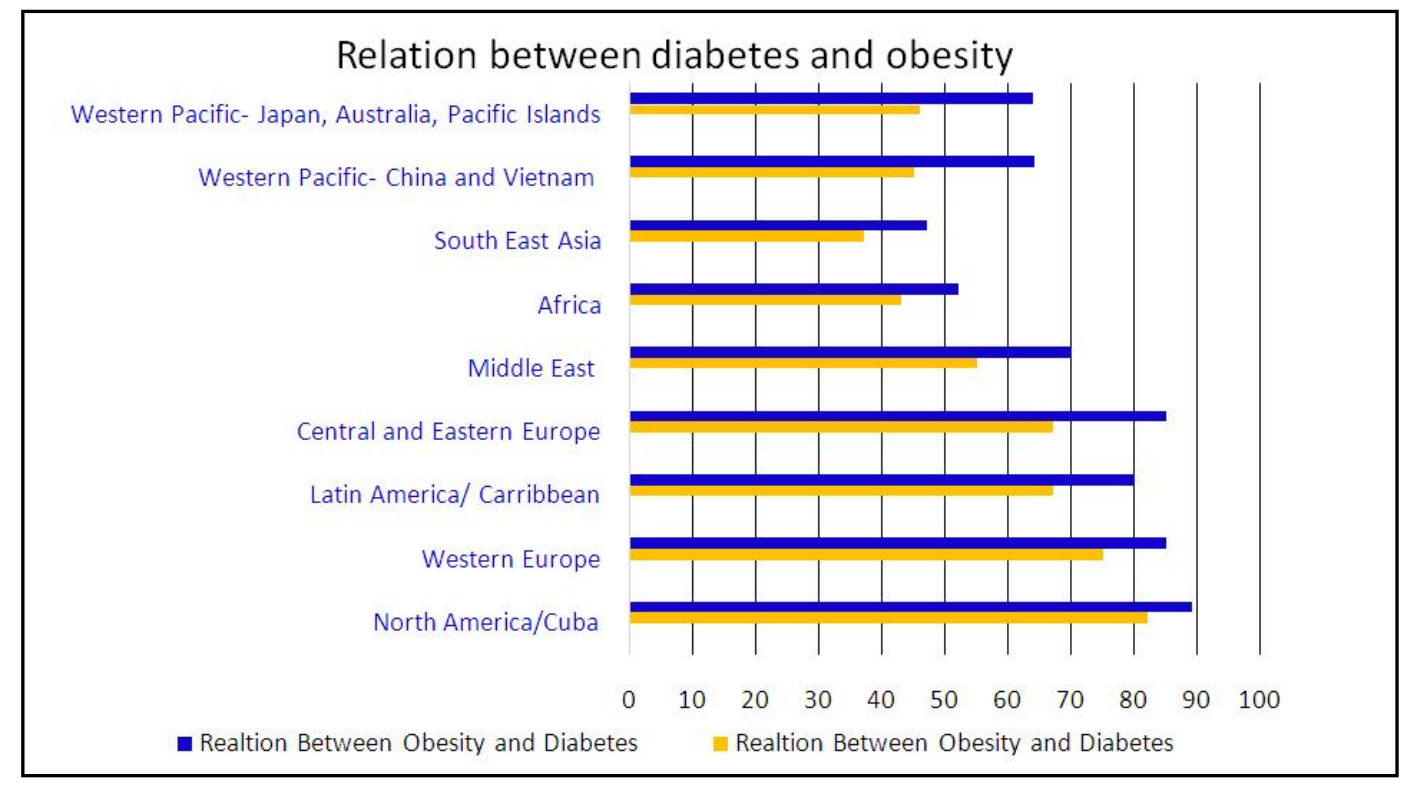

Figure 1: Relation between diabetes and obesity (Zhu and Zhang et al., 2016).

\subsection{Etiology of Type 1 diabetes mellitus}

Type 1 diabetes is an ongoing issue wherethe obliteration of pancreatic beta cells happens, bringing about insulin inadequacy. Most of the cases are because of an immune system intervened annihilation of beta cells which is called type $1 \mathrm{a}$ and a little minority of cases results from an idiopathic obliteration of beta cells which is called type $1 \mathrm{~b}$. Type 1 diabetes is likewise the most normal sort of diabetes.

Type 1 diabetes can be analyzed at whatever stage in life yet a greater part of the time it is appeared to happen at age of 5-7 years or close to pubescence. It is supposed to be more inclined to men and young men instead of in ladies. All around the world, the commonness of type 1 diabetes fluctuates significantly (Liese et al., 2006). Type 1 diabetes is generally basic in Finland, there are $>60$ cases per 100000 individuals every year, and Sardinia around 40 cases for every 100000 individuals every year. On the other hand, the problem is exceptional in China, India, and Venezuela (around 0.1 cases per 100000 individuals every year). There is seen a great deal of variance in prevalence among Europe and North America. Ayearly expansion in the predominance of type 1 diabetes is additionally revealed in nations like Finland (2.4), Germany (2.6), and Norway (3.3). While there are likewise a few nations where the pace of rate of type 1 diabetes has been diminished throughout the long term like Sweden.

\section{Pathophysiological factors for gestational diabetes}

i. $\beta$-Cell dysfunction: The primary role of $\beta$-cells is to store and secrete insulin to control the levels of glucose. When the capacity of beta-cells to maintain a proper glucose concentration is lost, this is classified as $\beta$-cell dysfunction (Sharma et al., 2001). The causes of beta-cell dysfunction are varied and complex. Defects can occur at any point during the manufacturing process: Pro-insulin synthesis, post-translational changes, granule storage, and blood glucose concentration sensing (De Fronzo et al., 2009). $\beta$-cell dysfunction is impaired by insulin resistance. Glucotoxicity is described as the direct contribution of glucose to beta-cell failure (Clark et al., 2017). 


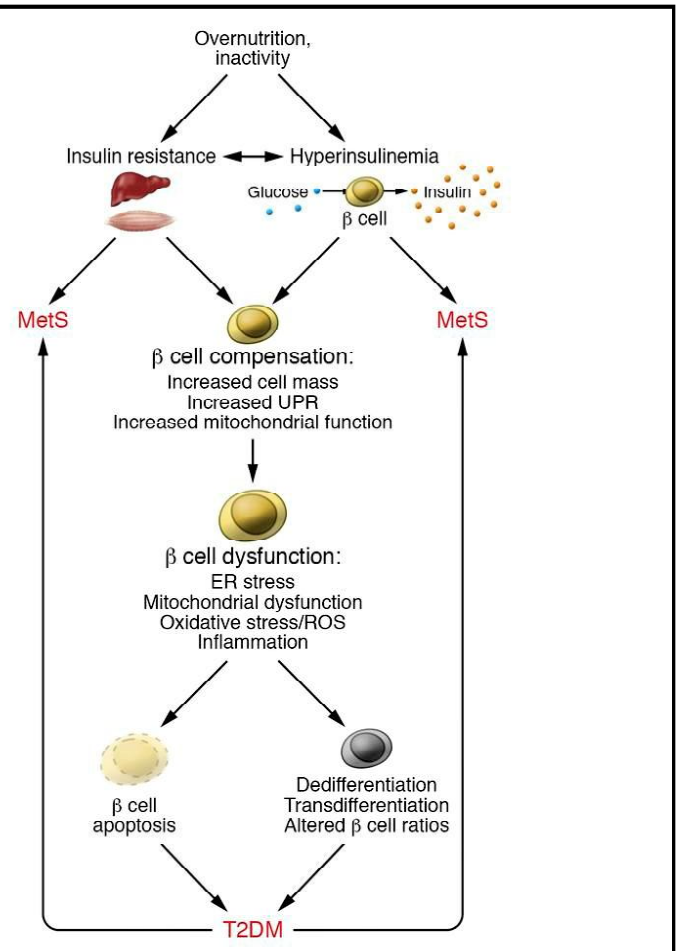

Figure 2: $\beta$ Cell compensation and dysfunction in MetS and T2DM (Laura et al., 2019).

A lifestyle of over nutrition and/or inactivity can give rise to an insulin-resistant condition and/or induce insulin hypersecretion from the pancreatic $\beta$ cell. In each case, A feedback loop can be set up to improve insulin secretion and intensify insulin resistance; each of these factors can lead to MetS and its drawbacks. Cells can functionally prepare for the elevated metabolic demand by increasing cell mass, triggering an unfolded protein response (UPR), and enhancing mitochondrial function at first. However, the stressed cells die, dedifferentiate, transdifferentiate, or change phenotypes, all of which compromise function. MetS may be exacerbated by disrupted cell activity (Figure 2) (Laura et al., 2019).

ii. Hepatic System: GDM is related to hepatic glucose production, known as gluconeogenesis. This condition is increased in the fasted state and inhibits in the fed state (Catalano et al., 2014). Common factors between insulin signalling and pathway controlpathways, such as phosphoinositide 3 kinase (PI3K), can play a role in these effects (Burks et al., 2001). The result of inaccurate glucose load because the majority of glucose absorption by the liver is not insulin-dependent, resulting in insulin resistance.

iii. Placental transport mechanism: Various factors of maternal, fetal, and origin of the placenta are controlled by placental function. Fetal signals influence the placental function including IGF II and PTHrp. The effects on maternal factors such as adipokines, hormones, and nutrient level have been studied in many models including cultured trophoblast cells, perfused placenta, placental explants. A variety of molecules that are released into maternal and fetal circulation are produced through the placenta. These compounds may have an autocrine and paracrine effect on the placenta. Placental nutrient transport is influenced by the integration of multiple, and often divergent, signalling by intracellular regulatory pathways such as mTOR, PPAR, and STAT (Figure 3) (Lager et al., 2012).

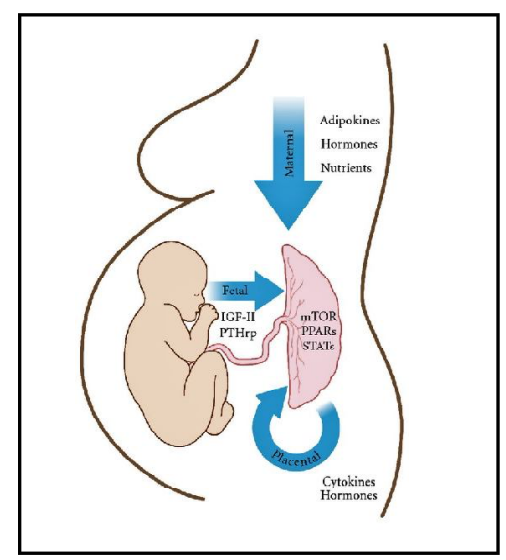

Figure 3: Maternal system's placental transport (Lager et al., 2012).

Placental glucose transportation facilitated diffusion is used to transfer glucose from the placenta to the bloodstream. During pregnancy, the placenta contributes to insulin resistance utilizing the emission of hormones as well as cytokines. The placenta serves as a shield between the mother and the fetus, exposed itself to high levels of blood glucose (hyperglycemia). This can affect glucose, amino acids, and lipid transport through the placenta.

iv. Glucose: Glucose is a source of nutrition for the fetus and placenta. Insulin is not necessary for glucose transport in the placenta. Transport of glucose is done by means GLUT1, by a carrier-mediated sodium independent diffusion (Augustin et al., 2010). The placenta communicates the insulin receptors and insulin signalling which can influence glucose metabolism in the placenta. However, glucose uptake in the placenta means the maternal body is sensitive to hyperglycemia and this contributes to increased fetal growth known as macrosomia (Lang et al., 2006).

Table 1: Classification of diabetes mellitus (Stevens et al., 1995)

\begin{tabular}{|l|ll|}
\hline \multicolumn{1}{|c|}{ Classification } & \multicolumn{1}{|c|}{ Pathogenesis } \\
\hline \multirow{2}{*}{ 1. Type I } & $\bullet$ & Destruction of beta cells \\
\cline { 2 - 3 } & $\bullet$ & Deficiency of insulin \\
\cline { 2 - 3 } & $\bullet$ & Autoantibodies of insulin \\
\cline { 2 - 3 } & $\bullet$ & IA-2 and IA2B autoantibodies \\
\cline { 2 - 3 } & $\bullet$ & Autoantibodies of Islets cells \\
\hline 2. Type II & $\bullet$ & $\begin{array}{l}\text { Insulin resistance with an insulin } \\
\text { secretary }\end{array}$ \\
\hline \multirow{2}{*}{ 3. Gestational } & $\bullet$ & Deficiency of insulin in the body \\
\cline { 2 - 3 } & Diabetes & $\begin{array}{l}\text { Changes due to hormonal and } \\
\text { metabolic during pregnancy }\end{array}$ \\
\cline { 2 - 3 } & $\bullet$ & $\begin{array}{l}\text { Glucose intolerance with onset during } \\
\text { pregnancy }\end{array}$ \\
\hline
\end{tabular}




\subsection{Pathophysiological factors for Type 1 diabetes mellitus}

Whenever somebody takes the meal, there is a rise in blood glucose levels that stimulates insulin secretion resulting in an increase in transportation, biotransformation, and storage in muscles and fat tissues. In fasting conditions, the glucose in the blood is provided by the liver that is used by the brain, without any dependency on insulin. Besides, the storage of glucose, insulin also inhibits the secretion of glucagon and lowers the concentration of serum fatty acids leading to a decline in liver glucose production (Kangralkar $e t$ $a l ., 2010)$. Insufficient insulin or resistance to insulin in the body results in reduced tissue uptake of glucose that results in intracellular hypoglycemia and extracellular hyperglycemia. The intracellular hypoglycemia causes glycogenesis and gluconeogenesis that leads to fats breakdown (causing diabetic ketoacidosis) and decreases protein synthesis and gamma globulins (causing cachexia, polyphagia, and impaired wound healing), while the extracellular hyperglycemia leads to hyperglycaemic coma and osmotic dieresis (Ozougwu et al., 2013).

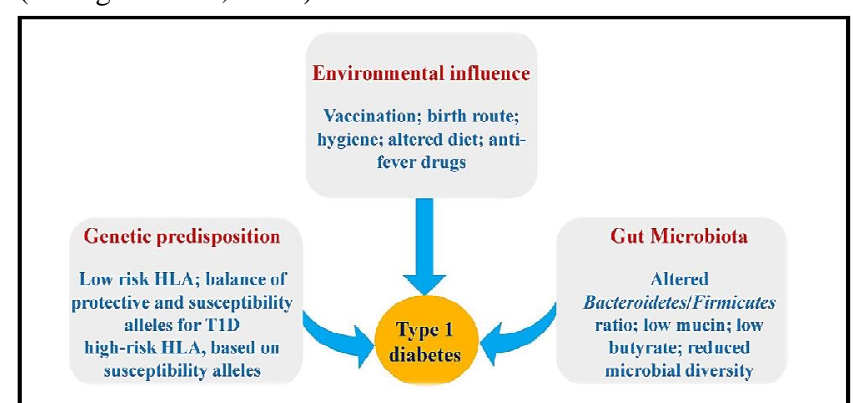

Figure 4: Type 1 diabetes mellitus susceptibility (T1D) factors (Rewers et al., 2018).

In the development of $\mathrm{T} 1 \mathrm{D}$, genetic predisposition, in combination with environmental factors, is critical. T1D is characterized by an increase in autoantibodies against cell antigens, as well as insulitis (pancreatic inflammation), which is accompanied by insulin secretion reduction and cell death. The exact environmental causes that cause T1D pathogenesis are unknown; however, genetics and/or the environment are two of the most frequently implicated factors. Viral diseases, antibiotics, early ingestion of cereal or cow-milk proteins, reduced vitamin D intake or endogenous synthesis, lack of breastfeeding, seasonality exposure to endocrine-disrupting chemicals (Figure 4) (Rewers et al., 2018).

In insulin-dependent diabetes mellitus (IDDM) insulin secretion is deficient due to the autoimmune destruction of beta-pancreatic cells that leads to metabolic disturbances associated with IDDM (Ozougwu et al., 2013). The end-stage of beta-cell destruction represents the onset of clinical disease leading to type 1 diabetes mellitus, in which there are infiltrate-ing monocytes, lymphocytes, and a mixture of pseudomorphic islets with some cells secreting somatostatin, glycogen, and pancreatic polypeptide which then, consequently through the immunogenic process, induces the disease (Homsi et al., 1993) (Yagi et al., 1992). Autoimmunity, genetic makeup, and environmental factors are responsible for is let cell destruction (Michael et al., 2000).

\section{Biomarkers for diabetes (T1DM and GDM) screening}

Biomarkers are defined as medical signs that help us to observe the medical condition of a patient from the outside. The use of biomarkers has become very common in clinical research. A lot of biomarkers are also used to predictive and diagnostic biomarkers are used to predict GDM. GDM mostly occurs in the $2^{\text {nd }}$ trimester of pregnancy with increased insulin resistance, inflammation, betacell disruption. In some early stages of complicated pregnancies some proteins, miRs are released into blood and urine and these can serve as biomarkers for GDM.

i. Adipose tissue-derived markers: Adipose tissue is an endocrine organ. It releases adiponectin which contains antiatherogenic, anti-inflammatory, and insulin-sensitizing properties. Adiponectin is inversely associated with obesity, hypertension, serum lipids, and coronary artery disease. Adiponectin is also said to be decreased during pregnancy, if the insulin level also decreases (Arita et al., 2012). Several studies have addressed adiponectin to be an early indicator of GDM. Adiponectin therapy has been tested in animal models of obesity and it has shown that we can improve glycemia without alterations in body weight. Adiponectin can also be important for the pathophysiology of GDM (Hedderson et al., 2013).

Leptin is an adipocyte hormone that helps to regulate energy through hypothalamic pathways and is produced in adipocytes as wells as ovaries and placenta (Wauters et al., 2000). In contrast to adiponectin, leptin level increases in pregnant women due to placental secretion, and these increased levels are mostly observed in women with GDM. But, in recent studies, it is considered a poor predictor of GDM (Briana et al., 2009).

ii. Placenta secreted biomarkers: Visfatin has endocrine, paracrine, and autocrine actions and is produced by visceral fat. In recent studies, it is observed that in the second trimester of pregnancy, the level of visfatin increases and then decreases in the third trimester. But, in some other studies, the increased rate of visfatin when added with other maternal risks, the detection rate of GDM did not increase. Visfatin is still considered a biomarker of GDM, but more studies and researches are still needed to be done regarding this (Filippatos et al., 2008).

iii. Inflammatory biomarkers: TNF- $\alpha$ is a proinflammatory biomarker and is produced by monocytes and macrophages. It affects the secretion and sensitivity of insulin by impairing B-cells function and insulin signalling pathways which result in insulin resistance and GDM. In recent studies, the increased level of TNF- $\alpha$ is observed in GDM patients. The increased level of TNF- $\alpha$ is due to oxidative stress and inflammation. But, further studies are still being done to gain more information regarding this factor (Cawthorn et al., 2008).

The acute phase protein high-sensitivity C-reactive protein (hsCRP) is produced primarily in tissue injury, inflammation, and infection. It is seen that an increased level of CRP in the first trimester is related to GDM risk (Wolf et al., 2003). After studying women with CRP in the highest quartile had a 3.5-fold increased risk of GDM relative to those in the lowest quartile, regardless of pre-pregnancy BMI, family history of DM, or null parity. After adjusting the increased risk of GDM incidence among women in the highest fertile compared to the lowest fertile was 3.6 times higher at CRP sampling when age, race/ ethnicity, smoking, parity, blood pressure, and gestational age were all taken into account (Berggren et al., 2015). 
Sex hormone-binding globulin (SHBG) is a glycoprotein and is important for the regulation and transportation of sex hormones. SHBG secretion is inhibited by insulin and insulin-like growth factor. (Hu, et al., 2015). The serum SHBG levels in GDM patients were found to be lower than in normal pregnancies in a study. The levels of SHBG were found to be even lower in women treated with insulin. This might suggest that SHBG could help to differentiate or predict the women who will require insulin therapy (Kopp et al., 2001).

Some of the most common biomarkers for type 1 diabetes are:

i. Genetic biomarkers: Genetic elements play a significant part in type 1 diabetes (T1D) pathogenesis. Although, linkage examines have uncovered the significant commitment of major histocompatibility complex (MHC) to T1D, most non-MHC hazard loci (like insulin (INS), cytotoxic T lymphocyte antigen 4 (CTLA4), protein tyrosine phosphatase, non-receptor type 22 (PTPN22), and interleukin 2 receptor alpha (IL2RA) were distinguished as important biomarkers (Barratt et al., 2004).

The human leukocyte antigen (HLA) qualities were the soonest and significant discoveries in the different parts in insusceptible response, for example, $\mathrm{T}$ cell determination and antigen introduction, HLA qualities can impact illness hazard and movement from various perspectives and can be viewed as the principal designated spot in the choice and actuation of autoimmunity field of T1D hereditary qualities. These qualities represent roughly half of the family grouping. INS and IL2RA are major T1D autoantigen and PTPN22 encodes the lymphoid protein tyrosine phosphatase (Ueda et al., 2003). Interferon induced with helicase $\mathrm{C}$ domain 1 (IFIH1) also takes part in natural insusceptibility through the acknowledgment of the RNA genomes of picornaviruses, giving a possible connection between hereditary defencelessness and ecological triggers since one of the proposed natural triggers of $\mathrm{T} 1 \mathrm{D}$, coxsackievirus $\mathrm{B} 4$, has a place with the picornavirus family (Liu et al., 2009).

ii. Transcriptomic biomarkers: The powerful condition of the transcriptome during prediabetes, illness movement, and clinical treatment can give possible biomarkers to sickness hazard expectation, infection subtype grouping, and helpful observing. Microarray innovation and bioinformatics permit the investigation of the entire transcriptome (quality articulation profile) in a solitary test.

Various examinations have endeavoured to find changes in quality articulation profiles during T1D improvement. The majority of these qualities are additionally upregulated in prediabetes subjects, recommending that they might be valuable prescient markers (Huang et al., 2003).

iii. Proteomic biomarkers: Protein levels execute the atypical hereditary and genomic changes, and along these lines are all the more straightforwardly related to cell capacity and wellbeing status. Hence, they have a more prominent potential to be utilized as biomarkers for sickness forecast or remedial observing. Today, the most helpful proteomic biomarkers for T1D (Wentzler et al., 2008). The four significant autoantibodies of clinical and examination interest are islet cell cytoplasmic autoantibodies (ICA), glutamic corrosive decarboxylase autoantibodies (GADA), insulin 2-related autoantibodies (IA2A), and insulin autoantibodies (IAA). ZnT8A, a recently perceived $\mathrm{ZnT}$ 8 islet autoantibody, may additionally improve the estimation of islet autoantibody testing (Orban et al., 2009). Undeniably, the danger of creating T1D will ascend with the expansion in the number of islet autoantibodies present. The danger for T1D in people with no autoantibody is just $0.5 \%$ and ascend to around $3 \%$ in people with one autoantibody. In subjects with two autoantibodies, the danger continues to ascend to $16 \%$, and leaps to $40 \%$ and half in subjects with three and four autoantibodies, separately. IAA is less prescient of T1D than different autoantibodies. Higher ICA titters and higher groupings of GADA were more impressive indicators of T1D than lower titters and lower focuses. Despite the utilization of the autoantibodies in the T1D forecast, they have a few genuine constraints (Burbelo et al., 2012).

Expanding endeavours have been given to novel T1D biomarker revelation over the most recent thirty years. To beat these issues, enhancements in advances and study configuration should be made in future investigations.

\section{Complications for gestational diabetes mellitus (GDM)}

GDM is described by hyperglycemia that develops during pregnancy and is triggered or exacerbated by underlying mechanisms such as genetic predisposition, insulin resistance, and chronic inflammation.

i. Maternal complications: In GDM, hyperglycemia may damage endothelial cells associated with hypertension results in vascular dysfunction (Stanley et al., 2011). During pregnancy and the postpartum period, GDM raises blood pressure (Shankar et al., 2010). Diabetes and hypertension are also risk factors for preeclampsia, a condition that affects pregnant women by 3-5\% of pregnancies worldwide. During pregnancy, hypertension prolonged insulin resistance and-cell dysfunction, which normally resolves after delivery (Powe et al., 2011), (Weissgerber et al., 2015).

ii. Fetal complications: The developing fetus has a limited ability to produce glucose; therefore, it derives most of its glucose from maternal blood. Maternal glucose crosses the placenta, while maternal insulin does not (Kc et al., 2015). According to Pedersen's hypothesis, if maternal glucose levels are high and uncontrolled, the excess glucose transported through the placenta induces increased fetal insulin production. Excess nutrient storage results in enlargement of fetal, or macrosomia. Between $15 \%$ and $45 \%$ of GDM pregnancies result in macrosomia the majority of adiposity is distributed around the fetal abdomen and shoulders, raising the likelihood of shoulder dystocia and birth trauma in infants (Sovio et al., 2016).

iii. Childhood and adulthood complications: The association between GDM and hyperglycemia in the offspring is well established. Obesity is linked to an increased risk of metabolic disorders, such as cardiovascular disease and insulin resistance, in the offspring of mothers with GDM (Vohr et al., 2008). Blood pressure, adiposity, hyperglycemia, and BMI were all found to be substantially higher in children born to GDM mothers. GDM offspring are also $29 \%$ more likely to develop diabetes in CVDs that develop early in life, such as heart failure, hypertension, deep vein thrombosis, and pulmonary embolism (Tam et al., 2017) (Pathirana et al., 2020). 


\section{Screening and diagnostic criteria for GDM}

The screening test is performed to determine the potential health diseases or disorders in people who may not have any symptoms of the same to avoid future risks. Likewise, the screening test for GDM is done to detect the presence of increased risk of hyperglycemia (high blood glucose level) to prevent the adverse maternal (during pregnancy) and perinatal (from pregnancy till as long as a year after the birth) outcomes and also the risk of future Diabetes predominantly type 2 (Gupta et al., 2015).

Screening for GDM is usually done at 24-28 weeks of gestation since resistance to insulin increases in the second trimester of pregnancy which leads to higher levels of glucose in women who cannot produce enough insulin to adopt this resistance (Rani et al., 2016). Gestational diabetes is asymptomatic, and therefore, the need for screening is essential (Akhalya et al., 2019). Two methods of screening that are in practice today are mentioned below:

i. Universal screening

ii. Selective screening

In the universal screening, all pregnant women are screened for GDM while in selective screening only those women are screened who show the presence of risk factors for gestational diabetes (Akhalya et al., 2019).
After a 7-year international epidemiological study of 23,325 pregnant women at 15 centers in nine countries known as the hyperglycemia and adverse pregnancy outcome study (HAPO), The International Association of Diabetes and Pregnancy Study Groups (IADPSG) panel decided on a one-step approach for the diagnosis (will be explained in detail in further section) which showed a significant increase in gestational diabetes diagnosis rates as well as higher primary cesarean delivery rates. Therefore, American Diabetes Association(ADA) recommended a new approach known as the two-step approach which is usually considered a better option (Akhalya et al., 2019). Hence, two methods proposed by ADA for the diagnoses of GDM in women without pre-existing diabetes are:

\section{i. One-step approach \\ ii. Two-step approach}

\subsection{One-step approach for the diagnoses of GDM}

It uses the protocol where a 75-g OGTT (Oral glucose tolerance test-diagnostic test) is performed which involves, obtaining a fasting plasma glucose after an overnight fast of $\geq 8$ hours, later administering glucose by ingestion (a liquid which should contain $75 \mathrm{~g}$ glucose), and again taking plasma glucose assessments after 1 and 2 hours. GDM is diagnosed if plasma glucose values equal or exceed (Rani et al., 2016).

Table 2: Diagnoses of GDM by one-step approach (Rani et al., 2016)

\begin{tabular}{|l|l|l|l|}
\hline Diagnosed & $\begin{array}{l}\text { Fasting plasma glucose } \\
\text { values equal or exceed }\end{array}$ & $\begin{array}{l}\text { 1-hour plasma glucose } \\
\text { values equalor exceed }\end{array}$ & $\begin{array}{l}\text { 2-hour plasma glucose } \\
\text { values equal or exceed }\end{array}$ \\
\hline Yes & $92 \mathrm{mg} / \mathrm{dl}(5.1 \mathrm{mmol} / \mathrm{l})$ & $180 \mathrm{mg} / \mathrm{dl}(10.0 \mathrm{mmol} / \mathrm{l})$ & $153 \mathrm{mg} / \mathrm{dl}(8.5 \mathrm{mmol} / \mathrm{l})$ \\
\hline
\end{tabular}

\subsection{Two-step approach for the diagnoses of GDM}

As the name suggests, this approach is divided into two stages: Prelims-GCT (glucose challenge test-screening test), mains- a 100g OGTT (oral glucose tolerance test), GCT measures the body's response to sugar (glucose) whereas 50-g GCT is performed which involves testing plasma glucose concentration after the consumption of $50 \mathrm{~g}$ glucose 1 hour later. If, the resultant value is $\geq 140 \mathrm{mg} / \mathrm{dl}$, then the patient is advised to proceed further with step- 2 i.e., mains and if does not exceeds then there is no need for the mains test (Rani et al., 2016) (Akhalya et al., 2019).

Step-2 uses the protocol where a $100 \mathrm{~g}$ OGTT is performed which involves obtaining a fasting plasma glucose, later administering glucose by ingestion (a liquid which should contain $100 \mathrm{~g}$ glucose), and again taking plasma glucose assessments after 1, 2, and 3 hours later. According to Carpenter and Coustan, GDM is diagnosed if PG values equal to or exceed (Rani et al., 2016).

- 1 hour PG is $\geq 10 \mathrm{mmol} / \mathrm{l}(180 \mathrm{mg} / \mathrm{dl})$

- 2 hours $P G$ is $\geq 8.6 \mathrm{mmol} / \mathrm{l}(155 \mathrm{mg} / \mathrm{dl})$

- 3 hours $P G$ is $\geq 7.8 \mathrm{mmol} / \mathrm{l}(140 \mathrm{mg} / \mathrm{dl})$

Also, according to NDDG (national diabetes data group), GDM is diagnosed, if PG values equal or exceed (Akhalya et al., 2019).

- fasting is $\geq 105 \mathrm{mg} / \mathrm{dl}$

- 1 hour is $\geq 190 \mathrm{mg} / \mathrm{dl}$

- 2 hours is $\geq 165 \mathrm{mg} / \mathrm{dl}$

- 3 hours is $\geq 145 \mathrm{mg} / \mathrm{dl}$

Table 3: Diagnoses of GDM by two-step approach

\begin{tabular}{|c|c|c|c|c|c|}
\hline Criteria & Diagnosed & $\begin{array}{l}\text { Fasting plasma } \\
\text { glucose values } \\
\text { equal or exceed }\end{array}$ & $\begin{array}{l}\text { 1-hour plasma } \\
\text { glucose values } \\
\text { equal or exceed }\end{array}$ & $\begin{array}{l}\text { 2-hour plasma } \\
\text { glucose values } \\
\text { equal or exceed }\end{array}$ & $\begin{array}{l}\text { 3-hour plasma } \\
\text { glucose values } \\
\text { equal or exceed }\end{array}$ \\
\hline $\begin{array}{l}\text { Carpenter } \\
\text { and coustan }\end{array}$ & Yes & $\begin{array}{l}5.3 \mathrm{mmol} / 1 \\
(95 \mathrm{mg} / \mathrm{dl})\end{array}$ & $\begin{array}{l}10 \mathrm{mmol} / \mathrm{l} \\
(180 \mathrm{mg} / \mathrm{dl})\end{array}$ & $\begin{array}{l}8.6 \mathrm{mmol} / \mathrm{l} \\
(155 \mathrm{mg} / \mathrm{dl})\end{array}$ & $\begin{array}{l}7.8 \mathrm{mmol} / \mathrm{l} \\
(140 \mathrm{mg} / \mathrm{dl})\end{array}$ \\
\hline $\begin{array}{l}\text { NDDG (national } \\
\text { diabetes data } \\
\text { group) }\end{array}$ & Yes & $105 \mathrm{mg} / \mathrm{dl}$ & $190 \mathrm{mg} / \mathrm{dl}$ & $165 \mathrm{mg} / \mathrm{dl}$ & $145 \mathrm{mg} / \mathrm{dl}$ \\
\hline
\end{tabular}




\section{Comparative study for screening and diagnostic of GDM and Type 1 diabetes}

Type 1 diabetes, which is predominantly due to autoimmunemediated destruction of pancreatic $\beta$-cells and results in absolute insulin deficiency (Atkinson et al., 2014). It can be diagnosed by any of the following methods:

i. Glycated haemoglobin (A1C) test: This type of blood test indicates the average blood sugar level for the past two to three months. It measures the percentage of blood sugar attached to the oxygen-carrying protein in red blood cells (haemoglobin). The more, the blood sugar level the more the haemoglobin has been attached with sugar. An A1C level of 6.5 per cent or greater indicates diabetes (Atkinson et al., 2014).

ii. Random blood sugar test: As the name suggests, a blood sample will be taken at any random time and may be confirmed by repeated testing. A random blood sugar level of $200 \mathrm{mg} / \mathrm{dl}$ $(11.1 \mathrm{mmol} / \mathrm{l})$ or higher suggests diabetes, specifically when coupled with any of the signs and symptoms of diabetes, likefrequent urination and extreme thirst (Atkinson et al., 2014).

iii. Fasting blood sugar test: In this test, a blood sample will be taken after an overnight fast. The table below shows various criteria.

Table 4: Diagnosis of Type 1 diabetes mellitus

\begin{tabular}{|l|l|}
\hline Diagnosed & Fasting blood sugar level \\
\hline No & $>100 \mathrm{mg} / \mathrm{dl}(5.6 \mathrm{mmol} / \mathrm{l})$ \\
\hline Prediabetes & $100-125 \mathrm{mg} / \mathrm{dl}(5.6$ to $6.9 \mathrm{mmol} / \mathrm{l})$ \\
\hline Yes & $126 / \mathrm{dl}(7 \mathrm{mmol} / \mathrm{l})$ or greater \\
\hline
\end{tabular}

If, the estimated value is $126 \mathrm{mg} / \mathrm{dl}(7 \mathrm{mmol} / \mathrm{l})$ or greater on two separate tests, we can say that one is having diabetes. Now, to recognize either the individual has type 1 or type 2 specialist may likewise run blood tests to search for autoantibodies that are regular in type 1 diabetes. These tests assist specialists with recognizing type 1 and type 2 diabetes when the finding is dubious. The presence of ketones in urine likewise recommends type 1 diabetes, as opposed to type 2 (Atkinson et al., 2014).

After the discussion on the screening of both type 1 diabetes and GDM, it can be observed that the criteria for the confirmation of two are completely different. According to the study, GDM is related to a higher danger to develop diabetes later on. Diabetes-related autoantibodies (AABs) have been recognized in a little rate (generally under $10 \%$ ) of ladies with gestational diabetes. The prevalence of an autoimmune or a non-autoimmune type of GDM relies upon a pregnant lady's hereditary proneness. GDM may work with the identification of ladies in danger of developing diabetes further down the road, and the presence of autoimmunity in GDM assists with assessing the risk of type 1 or type 2 diabetes in the future. Specifically, the danger of future T1D ascends with expanding thenumber of positive autoantibodies. The predominance in gestational diabetes of these immune system markers of type 1 diabetes (T1D) has been evaluated in numerous investigations, along with the danger of movement of AABs-positive GDM towards weakened glucose guideline after pregnancy (Incani et al., 2019).

\section{Conclusion}

From the whole discussion, it can be concluded that since the dawn of civilization, GDM is a chronic global health issue because its prevalence among women not only increases the risk of serious complications for the health of the mother but on her offspring also. It is any level of glucose intolerance that occurs strictly in a pregnant woman who does not have any past glucose intolerance experience. GDM should be considered as an important health issue since in the long-term both the mother and her children are at a high risk of developing T2DM later in life. In this review article, we have seen that GDM requires continuous medication and patient selfcare management. Also, the epidemiology of T1DM and GDM claim that these are ongoing health issue which has various risk factors associated and its prevalence has increased in recent years due to the various diagnostic and screening criteria. After reviewing all the related articles on GDM and T1DM, one important aspect which comes to mind is that the Indian population is diverse and variable, hence judging international criteria on the Indian population may not be conclusive. So, we need a further comparative study on different diagnostic criteria concerning pregnancy outcomes for GDM.

Although, the scanning and administration of GDM prove to be a source of contention. This will necessitate the requirement to further study and demonstrate the benefits of universal and selective screening as well as the treatment's effectiveness in lowering the risk of long and short-term complications. From the whole study, it can be concluded that to prevent the further prevalence of this chronic health disease it is a must to change the lifestyle.

\section{Acknowledgements}

This work is supported by the School of Bioengineering and Biosciences, Lovely Professional University, Jalandhar, Punjab.

\section{Conflicts of interest}

The authors declare that there are no conflicts of interest relevant to this article.

\section{References}

Al Homsi, M.F. and Lukic, M.L. (1992). An update on the pathogenesis of diabetes mellitus. Department of pathology and medical microbiology (Immunology Unit) faculty of medicine and health sciences, UAE University, Al Ain, United Arab Emirates.

Aly, T.A.; Ide, A.; Jahromi, M.M.; Barker, J.M.; Fernando, M.S.; Babu, S.R.; Yu, L.; Miao, D.; Erlich, H.A. and Fain, P.R. (2006). Extreme genetic risk for type 1A diabetes PNAS., 103:140-174.

American Diabetes Association, (2004). Diagnosis and classification of diabetes. Diabetes Care, 27(1):S5-S10.

Anna, V.; Van der Ploeg, H.P.; Cheung, N.W.; Huxley, R.R. and Bauman, A.E. (2008). Sociodemographic correlates of the increasing trend in the prevalence of gestational diabetes mellitus in a large population of women between 1995 and 2005. Diabetes Care, 31:2288-2293.

Ashcroft, F.M.; Rohm, M.; Clark, A. and Brereton, M.F. (2017). Is type 2 diabetes a glycogen storage disease of pancreatic $\beta$ Cells? Cell Me Table, 26; $17-23$.

Atkinson, M.A.; Eisenbarth, G.S. and Michels, A.W. (2014). Type 1 diabetes. The Lancet, 383(9911):69-82.

Augustin, R. (2010). The protein family of glucose transport facilitators: It's not only about glucose after all. IUBMB Life, 62:315-333. 
Beyan, H.; Riese, H.; Hawa, M.I.; Beretta, G.; Davidson, H.W.; Hutton, J.C.; Burger H.; Schlosser, M.; Snieder, H. and Boehm, B.O. (2012). Glycotoxin and autoantibodies are additive environmentally determined predictors of type 1 diabetes: A twin and population study. Diabetes, 61:11921198.

Blüher, M.; Michael, M.D.; Peroni, O.D.; Ueki, K.; Carter, N.; Kahn, B.B. and Kahn, C.R. (2002). Adipose tissue selective insulin receptor knockout protects against obesity and obesity-related glucose intolerance. Developmental Cell, 3(1):25-38.

Burks, D.J. (2001).White, M.F.IRS proteins and beta-cell function. Diabetes, 50(1):S140-S145.

Catalano, P.M.(2014). Trying to understand gestational diabetes. Diabetes Med., 31:273-281.

De Fronzo, R.A. (2009). From the triumvirate to the ominous octet: A new paradigm for the treatment of type 2 diabetes mellitus. Diabetes, 58:773-795.

Dharma Shankar, K. (2010). Widlansky, M.E. vascular endothelial function, and hypertension: Insights and Directions. Curr. Hypertens. Rep., 12:448-455

Gupta, Y.; Kalra, B.; Baruah, M.P.; Singla, R.; and Kalra, S. (2015). Updated guidelines on screening for gestational diabetes. International Journal of Women's Health, 7:539

Hedderson, M. (2012). Racial/ethnic disparities in the prevalence of gestational diabetes mellitus by BMI. Diabetes Care, 35:1492-1498.

Hober, D. and Sauter, P. (2010): Pathogenesis of type 1 diabetes mellitus: interplay between enterovirus and host. Nature Reviews Endocrinology, 6:279-289.

Jaïdane, H.; Sauter, P.; Sane, F.; Goffard, A.; Gharbi, J. and Hober, D. (2010) Enteroviruses and type 1 diabetes: Towards a better understanding of the relationship. Reviews in Medical Virology, 20:265-280.

Jansson, T. and Powell, T.L. (2010). Role of the placentain fetal programming: Underlying mechanisms and potential interventional approaches. Clin. Sci. Lond. Engl., 113:1-13.

Joshi, S.R.; Parikh, R.M. and Das, A.K. (2007). Insulin history, biochemistry, physiology, and pharmacology. J. Assoc. Phys. India, 55:19-23.

Kangralkar, V.A.; Patil, S.D. and Bandivadekar, R.M. (2010). Oxidative stress and diabetes: A review. Int. J. Pharm. Appl., 1(1):38-45.

Laura, I.; Hudish, Jane E.B. and Reusch, Lori Sussel (2019). $\beta$ Cell dysfunction during progression of metabolic syndrome to type 2 diabetes $\mathrm{J}$. Clin. Invest., 129(10):4001-4008.

Liese, A.D.; D'Agostino, R.B. and Hamman, R.F. (2006). The burden of diabetes mellitus among US youth: Prevalence estimates from the SEARCH for diabetes in youth study. Peds., 118:1510-1518.

Maritim, A.C.; Sanders, A.; and Watkins, Iii, J.B. (2003). Diabetes, oxidative stress, and antioxidants: A review. Journal of Biochemical and Molecular Toxicology, 17(1):24-38.

Ozougwu, J.C.; Obimba, K.C.; Belonwu, C.D. and Unaka lamba, C.B. (2013). The pathogenesis and pathophysiology of type 1 and type 2 diabetes mellitus. Journal of Physiology and Pathophysiology, 4(4):46-57.

Pathirana, M.M.; Lassi, Z.S.; Roberts, C.T. and Andraweera, P. (2020). Cardiovascular risk factors in offspring exposed to gestational diabetes mellitus in utero: Systematic review and meta-analysis. J. Dev. Orig. Health Dis., 14:29-37.
Powe,C.E.; Levine, R.J. and Karumanchi, S.A. (2014). Preeclampsia, a disease of the maternalendothelium: The role of antiangiogenic factors and implications for later cardiovascular disease. Circulation 2011, 123:2856-2869. Pro-inflammatory chemokines in human pancreatic islets. Diabetologia; 57:502-511.

R. Zhao, L. Xu, M.L. Wu, S.H. and Huang, X.J. Cao (2018). Maternalprepregnancy body mass index, gestational weight gaininfluence birth weight Women Birth, 31(1):e20-e25.

Rani, P.R. and Begum, J. (2016). Screening and diagnosis of gestational diabetes mellitus, where do we stand. Journal of clinical and diagnostic research. JCDR, 10(4):QE01.

Rewers, M.;Hyoty, H.; Lernmark, A.; Hagopian, W.; She, J.X.; Schatz, D.; Ziegler, A.G; Toppari, J.; Akolkar, B. and Krischer, J. (2018). The environmental determinants of diabetes in the young (TEDDY) Study: 2018 Update. Curr. Diabetes Rep., 18:136.

Vyakaranam, S.; Vanaparthy, S.; Nori, S.; Palarapu, S. and Bhongir, A.V. (2014). Study of insulin resistance in subclinical hypothyroidism Int. J. Health Sci. Res., 4(9):147-153.

Solomon, C. G. (1997). A prospective study of pregravid determinants of gestational diabetes mellitus. JAMA, 278:1078-1083.

Soltesz, G; Patterson, C.C. and Dahlquist, G. (2007). Worldwide childhood type 1 diabetes incidence what can we learn from epidemiology? Pediatr Diabetes, 8(6):6-14.

Sovio, U.; Murphy, H.R. and Smith, G.C.(1997). Accelerated fetal growth prior to diagnosis of gestational diabetes mellitus: A prospective cohort study of nulliparous women. Diabetes Care, 39:982-987.

Stanley, J.; Cheung, C.C.; Rueda Clausen, C.; Sankaralingam, S.; Baker, P.N. and Davidge, S.T. (2016). Effect of gestational diabetes on maternal artery function. reprod. Sci., 18:342-352.

Stevens A. and Lowe J. (1995). In pathology, mosby, London, pp:504-507.

Susanne Lager, Theresa L. Powell, (2012). "Regulation of nutrient transport across the placenta", Journal of Pregnancy, Article ID179827, pp: 14 .

Tam, W.H.; Ma, R.C.; Ozaki, R; Li, A.M.; Chan, M.H.M.; Yuen, L.Y.; Lao, T.T.H.; Yang, X; Ho, C.S. and Tutino, G.E. (2017). In utero exposure to maternal hyperglycemia increases childhood cardiometabolic risk in offspring. Diabetes Care, 40:679-686.

Vohr, B.R. and Boney, C.M. (2008). Gestational diabetes: Thefore runner for the development of maternal and childhood obesity and metabolic syndrome? J. Matern. Neonatal Med., 21:149-157.

Shuang, W.; Huixia Zhonghua, Y. and Fu Chan KeZaZhi, (2014). Analysis of the effect of risk factors at gestational diabetes mellitus. Reviews, 49(5):321-324.

Wallace, J.I. (2004). Management of diabetes in elderly. Clin. Diabetes, 17:1.

Weir, G.C.; Laybutt, D.R.; Kaneto, H.; Bonner-Weir, S. and Sharma, A. (2001). Betacell adaptation and decompensation during the progression of diabetes. Diabetes, 50(1):S154-159.

Weissgerber, T. and Mudd, L.M. (2015). Pre-eclampsia and diabetes. Curr. Diabetes Rep., 15:9579.

Zhu, Y. and Zhang, C. (2016). Prevalence of gestational diabetes and risk of progression to type 2 diabetes: A global perspective. Curr. Diab. Rep., 16:7. 